\title{
Effect of local anaesthetics on steroid-nicotinic acetylcholine receptor interactions in native membranes of Torpedo marmorata electric organ
}

\author{
H.R. Arias ${ }^{1}$, M.B. Sankaram ${ }^{2}$, D. Marsh ${ }^{2}$ and F.J. Barrantes ${ }^{1}$ \\ 'Instituto de Investigaciones Bioquimicas, Consejo Nacional de Investigaciones Cientificas y Tecnicas and Universidad Nacional del Sur, \\ Bahia Blanca (Argentina) and ${ }^{2}$ Max-Planck-Institut für biophysikalische Chemie, Abteilung Spektroskopie, Göttingen (F.R.G.)
}

(Received 19 February 1990)

Key words: Nicotinic acetylcholine receptor; Fluorescence quenching; Cholesterol; Spin label; ESR; (Torpedo marmorata )

Interactions between steroids and the nicotinic acetylcholine receptor (AChR) have been studied in native membrane vesicles from Torpedo marmorata electric organ by electron spin resonance (ESR) and fluorescence techniques. ESR spectra of spin-labelled cholestane (CSL) revealed that this steroid probe was incorporated into the AChR-rich membrane vesicles in regions which were to a certain extent enriched preferentially in the steroid, both in the presence and in the absence of local anaesthetics. Since the nitroxide group present in CSL is also a paramagnetic quencher of the intrinsic protein fluorescence, this property was used to characterize the AChR-steroid interactions. The quenching induced by CSL was sensitive both to AChR concentration and to the action of cholinergic agonists. In competition experiments, the ability of CSL to quench the AChR intrinsic fluorescence was markedly inhibited by benzocaine, tetracaine and QX-222 (a quaternary trimethylammonium derivative of lidocaine), and was totally inhibited by procaine. The effectiveness of local anaesthetics in inhibiting CSL-induced quenching followed the order: procaine $\gg$ benzocaine $\geq$ tetracaine $>$ QX-222. This inhibition effect was shown not to be charge-dependent. The data can be interpreted in terms of a model requiring specific association sites for local anaesthetics on the hydrophobic surface of the AChR which at least partially overlap with those for steroids.

\section{Introduction}

The nicotinic acetylcholine receptor (AChR) is a pentameric glycoprotein located in the postsynaptic membrane (for a review, see Ref. 1). All subunits form the wall of a cation-selective channel which is chemically gated by the neurotransmitter acetylcholine. The transient opening of the channel results in ion transport through the membrane. However, the persistent presence of agonist leads to desensitization of the AChR, a state associated with impaired ion conductance. Whether the lipid environment is able to modulate the function of the AChR has been the subject of various investigations (for a recent review, see Ref. 2).

\footnotetext{
Abbreviations: AChR, nicotinic acetylcholine receptor; ESR, electron spin resonance; Hepes, $N$-(2-hydroxyethyl)piperazine- $N^{\prime}$-2-ethanesulfonic acid; CSL, $4^{\prime}, 4^{\prime}$-dimethylspiro[5- $\alpha$-cholestane-3,2'-oxazolidin]-3'-yloxyl; CCh, carbamoycholine hydrochloride.

Correspondence: F.J. Barrantes, Instituto de Investigaciones Bioquimicas, Consejo Nacional de Investigaciones Cientificas y Tecnicas, Universidad Nacional del Sur, 8000 Bahia Blanca, Argentina.
}

Because of the high levels of cholesterol in the electric organ of Torpedinidae and native membranes isolated therefrom $[3,4]$, this steroid could potentially play a role in receptor function. There have been reports on the steroid and cholesterol requirements during solubilization and reincorporation of the $\mathrm{AChR}$ in order to maintain the agonist-induced state transitions [5]. When studying the effect of lipid composition on vesicle structure and AChR function, Criado et al. [6] found that a cholesterol analogue was necessary to mimic the kinetics of agonist-induced state transitions and for achieving maximal ion-flux responses. The enhancement of agonist-induced ion-fluxes by cholesterol in reconstituted systems $[7,8]$ has also been reported. Cholesterol depletion from AChR membranes increases the affinity of the receptor for agonist binding, concomitantly decreasing ion flux [9]. In reconstituted planar bilayers, cholesterol enhances the conductance and cooperativity of the ion channel [10]. There are at least two possibilities by which cholesterol exerts its effects on AChR functional properties: (1) by influencing the bulk physical properties of the membrane, or (2) by modulating AChR activity through association sites on the protein 
surface. The former possibility is suggested by the high endogenous cholesterol levels of native AChR-rich membranes [3,4]. The second possibility is suggested by electron spin resonance spectroscopy (ESR) data [1113], showing that spin-labelled androstanol, a steroid analogue, exhibits a high affinity for AChR. The results of labelling all AChR subunits by a photoreactive cholesterol analogue [14] lend further support to the second hypothesis. Jones and McNamee [15] have proposed the existence of nonannular sites for cholesterol.

Non-competitive blockers of the AChR are a heterogeneous group of compounds that includes aminated local anaesthetics. These compounds alter the steadystate agonist dose-response relationship without significantly altering the apparent dissociation constant. Three different mechanisms have been postulated to explain their blocking action: (1) plugging of the open channel, thus blocking ion translocation by steric hindrance (reviewed in Ref. 16); (2) allosteric conformational changes of the receptor involving channel closing and accelerated desensitization [17]; and (3) blocking of the physiological response by an indirect interaction via the lipids surrounding the receptor in the plasma membrane [18]. Heidmann et al. [19] have suggested two different types of sites for local anaesthetic substances: (a) a unique high-affinity site that is histrionicotoxin-sensitive; and (b) several low-affinity sites, which are histrionicotoxin-insensitive and are located at the lipid/ protein interface.

In this paper, we have studied the interaction between a spin-labelled steroid, local anaesthetics, and the AChR. We have taken advantage of the fact that the nitroxide group attached to the steroid nucleus exhibits paramagnetic quenching of the intrinsic protein fluorescence to characterize these interactions. Finally, we have used permanently charged molecules, such as QX222 (the trimethylammonium derivative of lidocaine), uncharged local anaesthetics, such as benzocaine, and molecules such as procaine and tetracaine, where the charge is $\mathrm{pH}$-dependent (cf. Fig. 1), to focus on the effect of net charges on the ligand-receptor interactions.

\section{Materials and Methods}

\section{Materials}

Torpedo marmorata specimens from the Bay of Arcachon, France, were generously provided by Prof. Dr. V. Whittaker (Max-Planck-Institut für biophysikalische Chemie, Göttingen, F.R.G.). The fish were maintained in aquaria for two months until use. $N$-[propionyl- ${ }^{3} \mathrm{H}$ ]Propionylated $\alpha$-bungarotoxin (spec. act. 107 $\mathrm{Ci} / \mathrm{mmol}$ ) was purchased from Amersham International, Buchs, U.K. DEAE-cellulose sheets (DE-81) were obtained from Whatman Inc. (Clifton, N.J.). Percoll (density $1.030 \mathrm{~g} / \mathrm{ml}$ ) was obtained from Pharmacia
(Uppsala, Sweden). The spin-labelled cholestane analogue $\left(4^{\prime}, 4^{\prime}\right.$-dimethylspiro[5- $\alpha$-cholestane-3, $2^{\prime}$-oxazolidin]-3'-yloxyl) was purchased from Syva (Palo Alto, CA). Procaine-, tetracaine- and carbamoylcholine-hydrochloride, and benzocaine were obtained from Sigma Chemical Co. (St. Louis, MO). QX-222 (a trimethylammonium derivative of lidocaine) was from Astra (Sweden). All other reagents were of the highest purity available.

\section{Methods}

Purification of AChR-rich membrane vesicles. The fish were killed by pithing, the electric organs were dissected and rapidly used for the preparation of AChR-containing membranes following the methods of Lindstrom et al. [20] or Barrantes [21]. Where indicated, sealed and leaky vesicles were further fractionated by the method of Sachs et al. [22], based on the exchange of $\mathrm{Na}^{+}$ within the vesicles for external $\mathrm{Cs}^{+}$and subsequent separation on a Percoll-CsCl density gradient. The specific activity of the AChR membranes was assayed using the $\alpha-\left[{ }^{3} \mathrm{H}\right]$ bungarotoxin/DE- 81 ion-exchange filter paper method [23]. Typically values of 900-1700 pmol $\alpha-\left[{ }^{3} \mathrm{H}\right]$ bungarotoxin $/ \mathrm{mg}$ protein were obtained. Protein was determined according to the method of Lowry et al. [24] using bovine serum albumin as standard. In the case of vesicles obtained by Percoll-CsCl density gradient, blanks containing Percoll-CsCl were subtracted.

ESR experiments. Sealed membrane vesicles were centrifuged in an Eppendorf centrifuge for $15 \mathrm{~min}$ at full speed in order to remove the remaining Percoll- $\mathrm{CsCl}$. The floating layer was washed with $10 \mathrm{mM}$ Hepes, $(\mathrm{pH}$ $8.0)$. The pellet $(0.3 \mathrm{mg}$ protein) was resuspended in 1 $\mathrm{ml}$ buffer and supplemented with $10 \mu \mathrm{g}$ CSL previously dissolved in absolute ethanol ( $1 \%$ of the total volume). Membrane and probe were allowed to interact at room temperature for $30 \mathrm{~min}$. In competition experiments, membranes were preincubated with different local anaesthetics for $30 \mathrm{~min}$. The membrane suspensions were subsequently incubated with CSL for an additional $30 \mathrm{~min}$ at a local anaesthetic/CSL molar ratio of $29.6,32.7$, and 53.9 for tetracaine, procaine, and benzocaine, respectively. Membrane samples were then centrifuged at $45000 \mathrm{rpm}$ in a Beckman $50 \mathrm{Ti}$ rotor for $45 \mathrm{~min}$ and the pellets were transferred to ESR sample capillaries ( $1 \mathrm{~mm}$ i.d.) and concentrated in a bench top centrifuge. To minimize the signal from water, the samples were trimmed to a height of $10 \mathrm{~mm}$ by carefully removing excess vesicle suspension and supernatant. ESR spectra were recorded with a Varian E-12 Century Line spectrometer equipped with a nitrogen gas-flow temperature regulation system. Temperatures were measured to $\pm 0.1^{\circ} \mathrm{C}$ with a thermocouple placed just above the cavity within the sample capillary. ESR spectra were collected using an IBM personal computer with a 
Labmaster interface (12 bit $\mathrm{A} / \mathrm{D}$ resolution) using software written by Dr. M.D. King (Max-Planck-Institut für biophysikallische Chemie) and stored as 1 kword data files. For further details of the ESR techniques, see Ref. 44.

Fluorescence measurements. For protein intrinsic fluorescence determinations $50-100 \mu \mathrm{g}$ AChR membrane protein (40-130 nM $\alpha-\left[{ }^{3} \mathrm{H}\right]$ bungarotoxin binding sites) were used. In all cases the membranes were resuspended by brief sonication (10 s) in $1 \mathrm{ml}$ of $100 \mathrm{mM}$ sodium phosphate buffer ( $\mathrm{pH}$ 8.0). To desensitize AChR, membranes were resuspended in the same buffer containing $100 \mu \mathrm{M} \mathrm{CCh}$ and incubated for $60 \mathrm{~min}$. To determine the steady-state fluorescence of the protein, membrane suspensions were placed in $1 \times 1,0.7 \times 0.7$, or $0.5 \times 1$ $(\mathrm{cm} \times \mathrm{cm})$ quartz cuvettes, excited at $285 \mathrm{~nm}$ and recorded in a spectrofluorimeter (SPF-500 Aminco-Bowman) at an emission wavelength of $340 \mathrm{~nm}$. The excitation and emission bandpass was $5 \mathrm{~nm}$. In the case of desensitization experiments, the fluorescence emission spectra of the protein were recorded between 300 and $400 \mathrm{~nm}$. The spectra were stored and their corresponding areas integrated using a Bascom-Turner Instrument (MA, U.S.A.). The fluorescence emission spectra and intensities of the local anaesthetics were determined in control experiments, at $300-400 \mathrm{~nm}$ with $285 \mathrm{~nm}$ excitation. In the particular case of procaine, increasing aliquots of the drug were added to the AChR suspension and the total fluorescence was measured at $340 \mathrm{~nm}$.

Quenchinq experiments. Aliquots of an isopropanol solution of CSL were added to the AChR suspension and allowed to interact for at least $1 \mathrm{~h}$ before fluorescence measurements. All values were corrected for dilution. In order to correct for trivial absorption from the CSL at the excitation and emission wavelengths used in the quenching experiments, the absorption of CSL was determined in buffer. The static absorption factor thus obtained, $I$, was used to correct the apparent quenching. The actual paramagnetic quenching by the nitroxide group covalently attached to the steroid nucleus was obtained from the formula:

$F_{\text {cor }}=F_{\text {app }} / I$

where

$I=10^{-A_{\mathrm{ex}}\left(L_{\mathrm{ex}} / 2\right)[\mathrm{C}] /\left[\mathrm{C}_{\mathrm{ex}}\right]} \cdot 10^{-A_{\mathrm{em}}\left(L_{\mathrm{em}} / 2\right)[\mathrm{C}] /\left[\mathrm{C}_{\mathrm{em}}\right]}$

and $F_{\text {cor }}$ and $F_{\text {app }}$ are the corrected and apparent fluorescence intensities after CSL quenching, respectively, $[C]$ is the concentration of CSL used in the fluorescence quenching experiments, and $\left[\mathrm{C}_{\mathrm{ex}}\right]$ and $\left[\mathrm{C}_{\mathrm{em}}\right]$ are the CSL concentrations used to determine absorption at the excitation $\left(A_{\mathrm{ex}}\right)$ and emission $\left(A_{\mathrm{em}}\right)$ wavelengths, respectively, and $L_{\mathrm{ex}}$ and $L_{\mathrm{em}}$ are the pathlengths of the cuvette along the excitation and emission axes, respec- tively. To minimize the CSL absorption at the excitation wavelength a $0.5 \times 1 \mathrm{~cm}$ cuvette was used with the 0.5 pathlength along the excitation axis.

Local anaesthetic treatment. AChR membranes were preincubated with local anaesthetic at room temperature or at $4^{\circ} \mathrm{C}$ for $1-2 \mathrm{~h}$ before addition of CSL. Of the local anaesthetics used in this study, only benzocaine and procaine exhibited a measurable intrinsic fluorescence in the same range as that of the AChR protein. The contribution of procaine and benzocaine fluorescence was determined as previously described, and subtracted from the observed total intensity.

\section{Results}

\section{ESR spectra of spin-labelled cholestane}

Fig. 1 shows the different local anesthetic compounds used in the present study.

The ESR spectra of CSL incubated with AChR-rich membrane vesicles in the presence and absence of various local anaesthetics are given in Fig. 2. The spectra are characteristic of anisotropic rotation of the molecule about its long axis, indicating that CSL is incorporated in the membrane. The ESR spectra are rather similar, both in the presence and absence of the different local anaesthetics. In all cases, the spectra exhibit a limited degree of spin-spin broadening, indicating that at least part of the spin-labelled steroid is preferentially concentrated in localized regions of the membrane. The same phenomenon has been observed for spin-labelled phosphatidylcholine and phosphatidylethanolamine [12], and most probably reflects an intrinsic property of the high packing density in the AChR-rich membranes. In the presence of benzocaine, this effect is even more pronounced.

As a result of the spin-spin broadening of the ESR spectra, it is difficult to resolve components in the wings

$$
\text { Benzocaine }
$$

Fig. 1. Molecular structures of the local anaesthetics used in this study. 
<smiles>CC(C)CCC1CCC2C3CCC4CC5(CCC4C3CCC12C)OCC(C)(C)N5O</smiles>

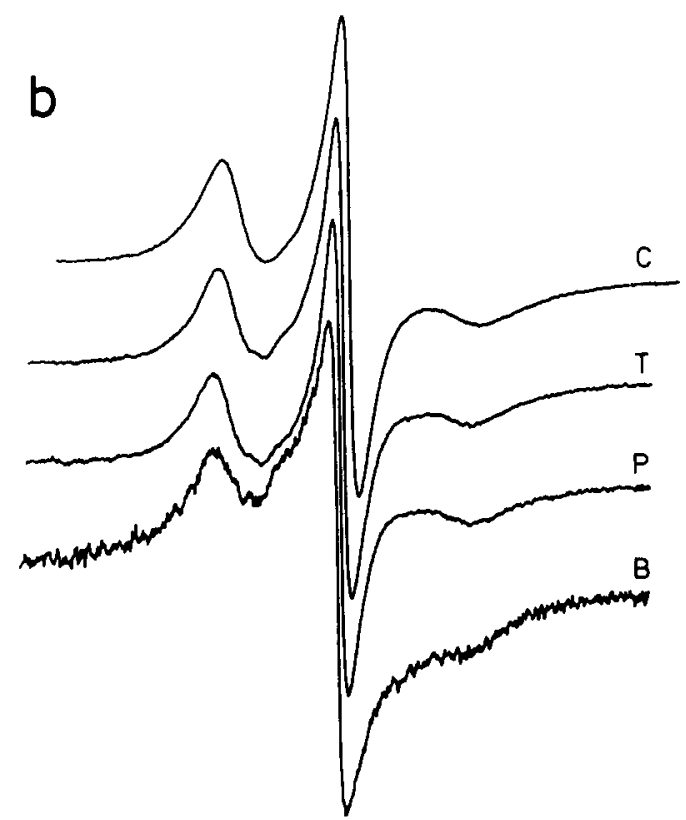

Fig. 2. (a) Molecular structure of spin-labelled cholestane (CSL). (b) ESR spectra of CSL in AChR-rich membrane vesicles from $T$. marmorata, at $22^{\circ} \mathrm{C}$. Native membranes $(\mathrm{C})$, were preincubated with procaine $(P)$, tetracaine $(T)$ and benzocaine $(B)$ for $30 \mathrm{~min}$ and then allowed to interact with CSL for $30 \mathrm{~min}$. Total scan width is 100 gauss.

of the spectra corresponding to CSL molecules that are motionally restricted by direct interaction with the AChR protein. However, comparison with previous results [11], indicates that CSL certainly does not exhibit the strong selectivity for the protein shown by spinlabelled androstanol or fatty acids. It is likely that its degree of association with the AChR is similar to, or smaller than, that of phosphatidylcholine or phosphatidylethanolamine [12]. In view of these uncertainties, it is not possible to deduce from the ESR spectra whether the local anaesthetics decrease the degree of association of CSL with the protein.

\section{Quenchinq of AChR intrinsic fluorescence spin-labelled cholestane}

Typical fluorescence emission spectra obtained with excitation at $285 \mathrm{~nm}$ are shown in Fig. 3. Upon addition of CSL to the native membrane from $T$. marmorata, the intrinsic fluorescence of the AChR protein was efficiently quenched by the nitroxide group attached to the CSL. Quenching parameters were obtained from these spectra according to the modified Stern-Volmer equation [45], which effectively assumes the existence of two fluorophore populations, one accessible and the other inaccessible to the quencher:

$F_{0} /\left(F_{0}-F\right)=1 /\left(f_{\mathrm{a}} K_{\mathrm{Q}}[\mathrm{CSL}]\right)+1 / f_{\mathrm{a}}$

where $F_{0}$ is the initial AChR fluorescence intensity, and $F$ is the fluorescence intensity of $\mathrm{AChR}$ in the presence of a given concentration, [CSL], of nitroxide. The value of $1 / K_{\mathrm{Q}}$, which can be defined as the CSL concentration at which $50 \%$ of the initial intensity is quenched assuming that all fluorophores are fully accessible to quencher, allows one to compare quantitatively the efficiency of quenching by CSL under different conditions. The other parameter characterizing the qenching is the apparent fraction of fluorophores, $f_{a}$, with effective quenching constant, $K_{\mathrm{Q}}$.

The analysis of the modified Stern-Volmer plots (Fig. 3 , inset), revealed that the fluorescence of AChR in the desensitized state was 4.7-fold more efficiently quenched by CSL than in the resting state (Table I).

Modified Stern-Volmer plots for CSL-induced quenching in membrane preparations containing different specific concentrations of AChR are given in Fig. $4 \mathrm{~A}$, and Table I summarizes the quenching data. The quenching efficiency with the membranes of higher specific activity, corresponding to $130 \mathrm{nM} \mathrm{AChR}$, was 5.4-fold higher than that with the lower specific activity membranes, which corresponded to $46 \mathrm{nM}$ AChR. The $f_{\mathrm{a}}$ and $K_{\mathrm{Q}}$ values summarized in Table I are plotted against AChR concentration in the resting state in Fig. 4B. The negative slope observed in the plot of $f_{\mathrm{a}}$ with increasing AChR concentration contrasts with the opposite trend found for the $K_{\mathrm{Q}}$ values.

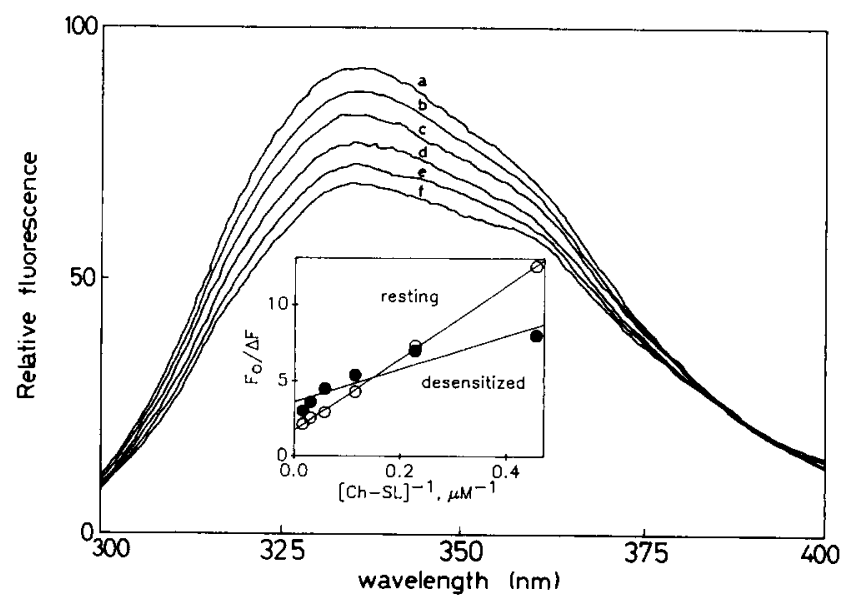

Fig. 3. (a) Fluorescence emission spectra of $120 \mathrm{nM}$ AChR-rich membranes from $T$. marmorata. (b-f) Quenching elicited by increasing concentrations of CSL. CSL concentrations were (in $\mu \mathrm{M}$ ): 2.2 (b), 8.8 (c), 17.6 (d), 33.0 (e) and 63.8 (f). (Inset) Modified Stern-Volmer plots for CSL quenching of intrinsic fluorescence from AChR in different conformational states. Temperature, $20^{\circ} \mathrm{C}$. 


\section{TABLE I}

Efficiency of spin-labelled cholestane in quenching the AChR intrinsic fluorescence

The apparent quenching parameters were obtained from the modified Stern-Volmer plots of Figs. 3 (inset) and 4A, where $f_{\mathrm{a}}=1$ /intercept is the apparent fraction of the fluorophores accessible to CSL; $K_{\mathrm{Q}}=$ intercept/slope, is the apparent Stern-Volmer quenching constant of the accessible fraction of fluorophores. $1 / K_{\mathrm{Q}}$, is the relative CSL concentration at which $50 \%$ of the fluorescence intensity is quenched if fluorophores are totally accessible to quencher $\left(f_{\mathrm{a}}=1\right)$. $r$, is the correlation coefficient of the linear regression. It is assumed that most of the AChR is in the resting state in the absence of agonist. The desensitized state is reached upon $60 \mathrm{~min}$ incubation of the membrane-bound AChR with $0.1 \mathrm{mM} \mathrm{CCh}$.

\begin{tabular}{lcccrr}
\hline $\begin{array}{l}\text { Conformational } \\
\text { state }\end{array}$ & $\begin{array}{l}\text { [AChR] } \\
\mathrm{nM}\end{array}$ & $f_{\mathrm{a}}$ & $\begin{array}{l}K_{\mathrm{Q}} \\
\left(\mathrm{M}^{-1}\right)\end{array}$ & \multicolumn{1}{c}{$\begin{array}{l}1 / K_{\mathrm{Q}} \\
(\mu \mathrm{M})\end{array}$} & $r$ \\
\hline Resting & 46 & 0.98 & $3.8 \cdot 10^{4}$ & 26.5 & 0.984 \\
Resting & 130 & 0.45 & $2.0 \cdot 10^{5}$ & 4.9 & 0.986 \\
Resting & 120 & 0.59 & $7.1 \cdot 10^{4}$ & 14.2 & 0.999 \\
Desensitized & 120 & 0.28 & $3.3 \cdot 10^{5}$ & 3.0 & 0.939 \\
\hline
\end{tabular}

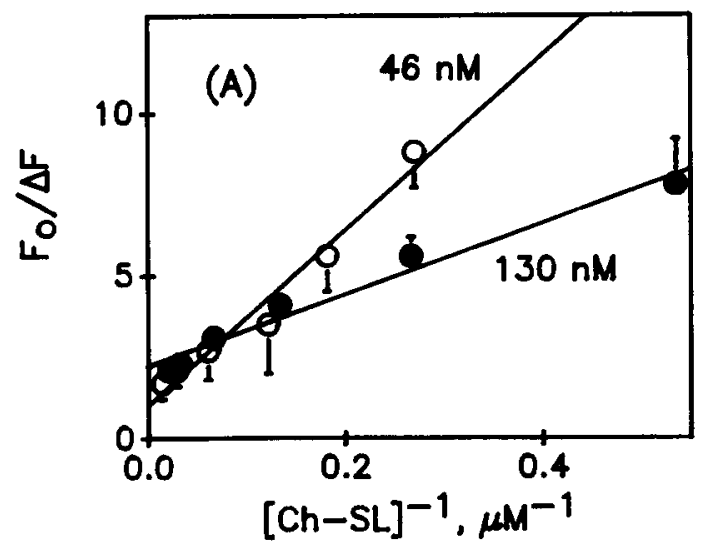

\section{TABLE II}

Effect of local anaesthetics on quenching of the AChR intrinsic fluorescence by spin-labelled cholestane

The apparent quenching parameters were obtained from the data of Figs. 5 and 6. Other details are given in the legend to Table I.

\begin{tabular}{lccccc}
\hline Anaesthetic & $\begin{array}{l}\text { Concn. } \\
(\mu \mathrm{M})\end{array}$ & $f_{\mathrm{a}}$ & $\begin{array}{l}\boldsymbol{K}_{\mathrm{Q}} \\
\left(\mathrm{M}^{-1}\right)\end{array}$ & \multicolumn{1}{c}{$\begin{array}{l}1 / K_{\mathrm{Q}} \\
(\mu \mathrm{M})\end{array}$} & $r$ \\
\hline None & - & 0.45 & $2.0 \cdot 10^{5}$ & 4.9 & 0.986 \\
QX-222 & 30 & 0.38 & $6.5 \cdot 10^{4}$ & 15.4 & 0.995 \\
Tetracaine & 30 & 0.31 & $5.0 \cdot 10^{4}$ & 19.8 & 0.991 \\
Benzocaine & 30 & 0.99 & $1.8 \cdot 10^{4}$ & 56.3 & 0.980 \\
Procaine & 3 & 0.99 & $2.6 \cdot 10^{4}$ & 38.0 & 0.998 \\
\hline
\end{tabular}

Fig. 5 compares the modified Stern-Volmer plots of AChR membranes pretreated with tetracaine, benzocaine and QX-222, respectively. The quenching parameters, summarized in Table II, demonstrate a lower efficiency of CSL-induced quenching upon exposure of

Fig. 4. (A) Modified Stern-Volmer plots for CSL quenching of intrinsic fluorescence from AChR membranes with different specific activities, corresponding to $46 \mathrm{nM}(O)$ and $130 \mathrm{nM}(\bullet)$ AChR sites. Temperature, $20^{\circ} \mathrm{C}$. (B) Fraction of accessible fluorophores $\left(f_{\mathrm{a}}\right.$, left scale) and quenching constant ( $K_{\mathrm{Q}}$, right scale) as a function of $\mathrm{AChR}$ concentration for a fixed membrane concentration.

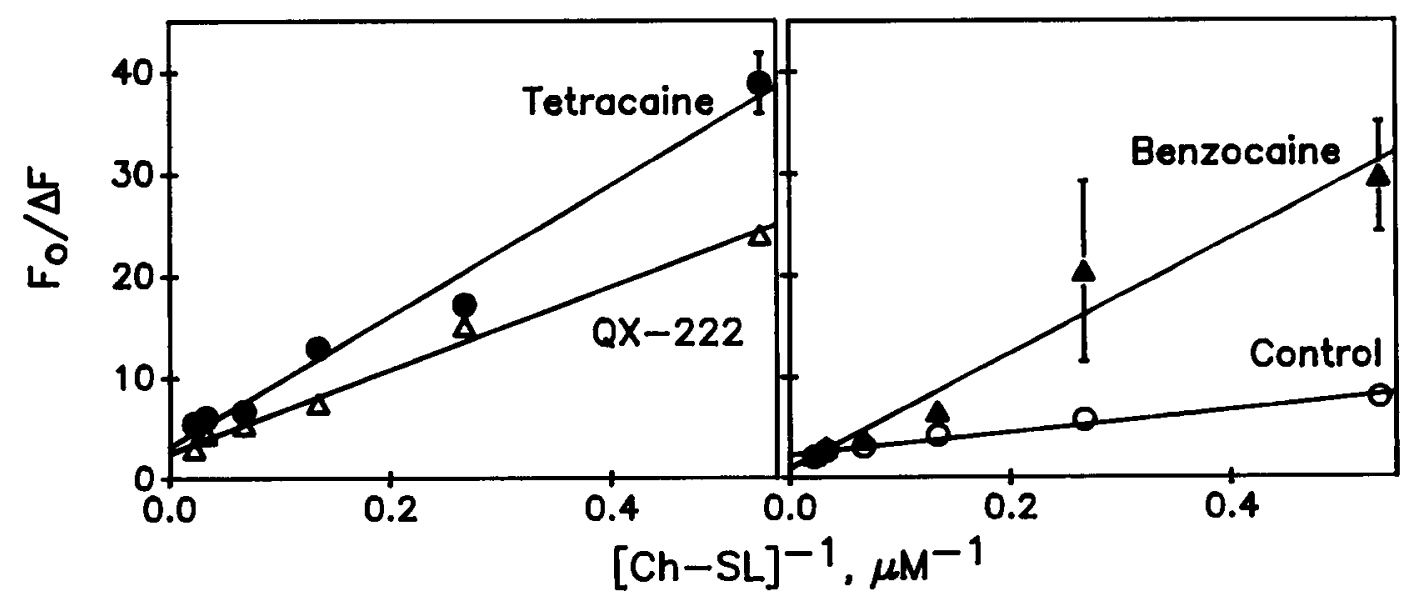

Fig. 5. Modified Stern-Volmer plots for CSL quenching of intrinsic fluorescence from AChR membranes pretreated with local anaesthetics. $130 \mathrm{nM}$ AChR membranes were preincubated, as described under Materials and Methods, with $30 \mu \mathrm{M}$ of the local anaesthetics indicated and then incubated with the given concentrations of CSL at $20^{\circ} \mathrm{C}$. 


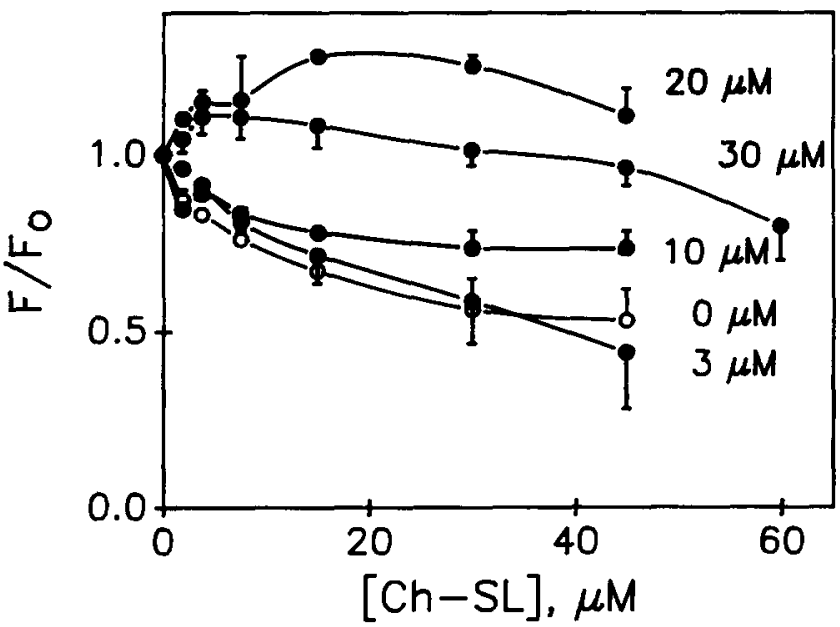

Fig. 6. Direct plots of the fraction of residual AChR fluorescence as a function of CSL concentration, after preincubation with procaine at the concentrations indicated. Temperature, $20^{\circ} \mathrm{C}$.

the AChR to local anaesthetics. The effects observed with procaine deserve special attention. Procaine inhibited completely the ability of CSL to quench the AChR fluorescence (Fig. 6).

These experiments demonstrate that the relative efficiency of local anaesthetics in inhibiting the CSL-induced quenching of $\mathrm{AChR}$ fluorescence follows the order: procaine $\gg$ benzocaine $\gtrsim$ tetracaine $>$ QX-222. Procaine was about 20 -fold more efficient than either QX-222 or tetracaine, and approximately 6-fold more efficient than benzocaine, in inhibiting quenching of AChR fluorescence by CSL.

\section{Discussion}

\section{Interactions between $C S L$ and the $A C h R$}

The fluorescence properties of the AChR in its native, membrane-bound state $[25,26]$, or in solubilized form [27], are typical of those found for integral membrane proteins. Fifty $\operatorname{Trp}$ and $80 \mathrm{Tyr}$ residues are present in Torpedo californica AChR [28]. The tryptophan residues dominate the fluorescence of the protein, but only one Trp is postulated to occur in the transmembrane region in both the 4- [28] and the 5-chain [29] AChR models. This residue may be accessible to quenching by CSL from the bilayer region, as was postulated to be the case with brominated lipids [15]. However, the nitroxide group of CSL is located close to the lipid/water interface [43], which most probably allows this spin label to quench fluorophores in the extramembranous domain of the AChR. This latter possibility is suggested by the relatively high levels of quenching $(37-57 \%)$ produced by CSL in native AChR-containing membranes. On the other hand, the complexity of the fluorescence from a multifluorophore protein such as the AChR [30] precludes over-detailed interpretations from simple quenching experiments.

Previous studies have shown that the intrinsic fluorescence of the $A C h R$ in native membranes is effectively quenched by spin-labelled fatty acids and androstanol $[11,26]$. Spin-labelled androstanol and CSL are steroid analogues which differ from the naturally occurring cholesterol either in the location or absence, respectively, of the steroid - $\mathrm{OH}$ group. It has been demonstrated that about $70 \mu \mathrm{M}$ spin-labelled androstanol is needed to inhibit $50 \%$ of the AChR intrinsic fluorescence [11]. The present results (Table I) show that CSL was between 2.5- and 13.6-fold more efficient than spin-labelled androstanol. This can be explained in terms of the different location of the paramagnetic nitroxide group relative to the membrane surface [43] and hence to the accessible tryptophan chromophores of the AChR [11]. In view of the high specificity of the androstanol analogue for the AChR [13], which it is clear from the ESR data presented here is not shared by the cholestane analogue (CSL), it is likely that the latter is the more appropriate analogue for studying the behaviour of the natural steroid, cholesterol.

The higher efficiency of CSL-induced quenching observed with desensitized $A C h R$ in comparison with resting AChR agrees with previous studies using spinlabelled stearic acid [11] and acrylamide [26] to assess the apparent accessibility of the AChR fluorophores from the lipid and aqueous phases, respectively, in the presence of the agonist suberyldicholine. Gonzalez-Ros et al. [31], however, have observed a lower accessibility of nitromethane to pyrene residues covalently attached to the $\mathrm{AChR}$, in the desensitized state. From the results presented here, we interpret the observed changes in CSL quenching efficiency upon addition of cholinergic ligands as being due to changes in the exposure of AChR fluorophores to quencher molecules accessing the AChR through the lipid matrix. Furthermore, the results suggest that the occupancy of agonist sites in the extracytoplasmic domain of the $\alpha$-subunits might induce local conformational changes with a relatively long time span (seconds to minutes), which are sensed over the quite considerable distances to the lipid/protein interface.

The lower fraction of accessible fluorophores $\left(f_{\mathrm{a}}\right)$ observed at higher relative $\mathrm{AChR}$ concentrations (Fig. 4B) suggests a phenomenon of fluorophore dilution. This could be explained by postulating that the sites at which CSL quenching takes place in the AChR present a lower number of fluorophores than in other non-receptor proteins present in the postsynaptic membrane. Thus, when the concentration of $\mathrm{AChR}$ is increased relative to other membrane proteins, the mean number of fluorophores diminishes. In contrast, quenching efficiency increases at higher specific AChR concentra- 
tions, suggesting a preferential interaction of CSL with association sites on the AChR (Fig. 4B).

\section{Effect of local anaesthetics on CSL quenchinq properties}

The fluorescence quenching method can be used to test the displacement of boundary lipids by other hydrophobic molecules such as fatty acids, cholesterol and local anaesthetics [32]. Thus, the question of whether local anaesthetics interact with AChR at steroid sites can be answered from the quenching properties of CSL in AChR membranes pretreated with local anaesthetics. Patch-clamp studies on myocytes of Xenopus laevis have demonstrated that the reduction in burst durations elicited by the general anaesthetic halothane was modulated by the level of cholesterol in the membrane [33].

Hille [34] has suggested that the pharmacological effect and rates of action of local anaesthetics depend on the relative population of uncharged/charged species. The neutral form would be more accessible to the excitable membrane than the cationic protonated species, thus explaining the faster rate of action of the former. Local anaesthetics that have tertiary amine headgroups, like procaine and tetracaine, may acquire a proton and a positive charge (see molecular structures in Fig. 1), depending on the $\mathrm{pH}$ of the medium. The intrinsic $\mathrm{pK}$ values of tertiary amines lie in the $\mathrm{pH}$ range 8.0-9.0. The interfacial $\mathrm{p} K$ of the membranebound form will be reduced by approx. $1 \mathrm{pH}$ unit, due to the lower polarity at the membrane surface [46]. Thus at the $\mathrm{pH}$ used here, the effect of tertiary amine local anaesthetics represents the sum of the populations of uncharged and charged molecules within the membrane, which is dominated by the neutral species.

The present results indicate that local anaesthetics compete with steroids for association sites on the AChR, with the following effectivity: procaine $\gg$ benzocaine $\gtrsim$ tetracaine $>\mathrm{QX}-222$. The order based on head-group charge at $\mathrm{pH} 8.0$, on the other hand, would be: QX-222 (permanently charged, trimethylammonium derivative of lidocaine) $\gg$ procaine (intrinsic $\mathrm{p} K$ 8.9) $>$ tetracaine (intrinsic $\mathrm{p} K \quad 8.2$ ) $>$ benzocaine (permanently uncharged). Thus, no simple relationship exists between net headgroup charge of the local anaesthetics and their effect on the steroid-AChR association sites.

Previous ESR experiments have dealt with charge effects on the interaction of spin-labelled local anaesthetics with the AChR, with somewhat diverse results. Thus, Earnest et al. [35,36] and Blanton et al. [37] have indicated that the positively charged form of spin-labelled intracaine gives rise to a higher proportion of the protein-associated species, whereas Horvath et al. [38], utilizing spin-labelled procaine and its thioester analogue, concluded that it is the uncharged form that has a greater degree of association with the protein. The former results are in agreement with the greater effectiveness of the charged cationic form of the drug in blocking receptor-mediated ion translocation measured by ${ }^{86} \mathrm{Rb}^{+}$flux [39], and with single-channel data on AChR [17] which suggest that local anaesthetic effects on AChR require that the molecule be positively charged. However, the latter ESR results [38] and the evidence that benzocaine, an uncharged local anaesthetic, blocks acetylcholine-activated ion channels [40], strongly suggest the opposite. No single model is therefore able to account wholely for the local anaesthetic effects.

\section{Relationship between local anaesthetic and steroid sites}

On the basis of the relative association constants determined by ESR, Horvath et al. [38] have divided the specificities of spin-labelled local anaesthetic analogues into three groups. Benzocaine, tetracaine, and procaine would be considered as drugs of high, medium, and low specificity, respectively, according to such a classification. In addition, the fraction of spin-labelled local anaesthetic analogues partitioning in the lipid phase follows the order: benzocaine $>$ procaine $\geq$ tetracaine, which corresponds approximately to the above pattern of specificity. This is in agreement with Koblin and Lester [18], who have previously reported that the effect of the more hydrophobic local anaesthetics are voltageindependent and have potencies that parallel their hydrophobicity. The fact that benzocaine has a greater effect on quenching by CSL than either tetracaine or QX-222 may be due to this property. On the other hand, the apparent equilibrium constant for the binding of benzocaine to its blocking site [40] is within a factor of five of those obtained for AChR-channel block by the charged drug QX-222 [17] and the partially ionized drug procaine [16]. This may also account for the greater effect of benzocaine in comparison to QX-222 on CSLinduced quenching. However, the lower specificity of spin-labelled procaine and the higher and approximately equivalent apparent equilibrium constants for benzocaine and QX-222, respectively, contrast strongly with the almost complete inhibition of the CSL-induced quenching by procaine. The data can be reconciled with a model postulating the existence of relatively specific sites for local anaesthetics on the hydrophobic surface of the AChR, which partially overlap with those for steroids. In the special case of procaine, the results strongly suggests a high degree of overlap.

In addition to the high affinity site for androstanol in the annular environment of the AChR [13], Jones and McNamee [15] have proposed the existence of nonannular sites for cholesterol at the interstices between the five AChR subunits. The existence of a putative hydrophobic path allowing uncharged local anaesthetics to interact with high affinity sites in the lumen of the AChR-channel has also been postulated [37,41,42]. An alternative mechanism of local anaesthetic action can thus be suggested, based on the intercalation of mole- 
cules in non-annular sites normally occupied by steroids in a hydrophobic corridor between AChR subunits. Such a corridor could provide a low affinity pathway to allow local anaesthetics to reach their high affinity sites inside the AChR channel. This suggestion is, however, highly speculative.

\section{Acknowledgements}

This work was supported by grants from the Consejo Nacional de Investigaciones Cientificas y Tecnicas (CONICET) and Fundación Antorchas, Argentina, and the Volkswagen Stiftung, F.R.G. H.R.A. was supported by a Deutscher Akademischer Austauschdienst e.V. (DAAD) fellowship.

\section{References}

1 Barrantes, F.J. (1988) in Physical properties of biological membranes and their functional implications (Hidalgo, C., ed.), pp. 147-175, Plenum Press, New York, London.

2 Barrantes, F.J. (1989) Crit. Rev. Biochem. Mol. Biol. 24, 437-478.

3 Gonzalez-Ros, J.M., Llanillo, M., Paraschos, A. and MartinezCarrion, M. (1982) Biochemistry 21, 3467-3473.

4 Rotstein, N.P., Arias, H.R., Barrantes, F.J. and Aveldano, M.I. (1987) J. Neurochem. 49, 1333-1340.

5 Criado, M., Eibl, H. and Barrantes, F.J. (1982) Biochemistry 21, 3622-3629.

6 Criado, M., Eibl, H. and Barrantes, F.J. (1984) J. Biol. Chem. 259, 9188-9198.

7 Dalziel, A.W., Rollins, E.A. and McNamee, M.G. (1980) FEBS Lett. 122, 193-196.

8 Fong, T.-M. and McNamee, M.G. (1986) Biochemistry 25, 830840.

9 Zabrecky, J.R. and Raftery, M.A. (1985) J. Receptor Res. 5, 397-417.

10 Schindler, H. (1982) in Transport in biomembranes: Model systems and reconstitution (Antolini, R., Gliozzi, A. and Gorio, A., eds.), pp. 75-85, Raven Press, New York.

11 Marsh, D. and Barrantes, F.J. (1978) Proc. Natl. Acad. Sci. USA $75,4329-4333$.

12 Marsh, D., Watts, A. and Barrantes, F.J. (1981) Biochim. Biophys. Acta 645, 97-101.

13 Ellena, J.F., Blazing, M.A. and McNamee, M.G (1983) Biochemistry $22,5523-5535$.

14 Middlemas, D.S. and Raftery, M.A. (1987) Biochemistry 26, 19 1223.

15 Jones, O.T. and McNamee, M.G. (1988) Biochemistry 27, 23642374.

16 Adams, P.R. (1981) J. Membr. Biol. 58, 161-174.

17 Neher, E. and Steinbach, J.H. (1978) J. Physiol. 277, 153-176.

18 Koblin, D.K. and Lester, H.A. (1979) Mol. Pharmacol. 15, 559_ 580.
19 Heidmann, T., Oswald, R.E. and Changeux, J.-P. (1983) Biochemistry 22,31123127 .

20 Lindstrom, J, Anholt, R., Einarson, B., Engel, A., Osame, M. and Montal, M. (1980) J. Biol. Chem. 255, 8340-8350.

21 Barrantes, F.J. (1982) in Neuroreceptors (Hucho, F., ed.), pp. 315-328, Walter de Gruyter \& Co., Berlin, New York.

22 Sachs, A.B., Lenchitz, B., Noble, R.L. and Hess, G.P. (1982) Anal. Biochem. 124, 185-190.

23 Schmidt, J and Raftery, M.A. (1973) Anal. Biochem. 52, 349-354.

24 Lowry, O.H., Rosebrough, N.J, Farr, A.L. and Randall, R.J. (1951) J. Biol. Chem. 193, 265-275.

25 Bonner, R., Barrantes, F.J. and Jovin, T.M. (1976) Nature 263 , $429-431$.

26 Barrantes, F.J. (1978) J Mol. Biol. 124, 1-26.

27 Kaneda, N., Tanaka, F., Kohno, M., Hayashi, K. and Yagi, K. (1982) Arch. Biochem. Biophys. 218, 376-383.

28 Noda, M., Takahashi, H., Tanabe, T., Toyosato, M., Kikyotani, S., Furutani. Y., Hirose, T., Takashima, H., Inayama, S., Miyata, T. and Numa, S. (1983) Nature 302, 528-532.

29 Finer-Moore, J. and Stroud, R.M. (1984) Proc. Natl. Acad. Sci. USA $81,155-159$.

30 Lakowicz, J.R. (1983) in Principles of fluorescence spectroscopy, Plenum Press, New York.

31 Gonzalez-Ros, J.M., Farach, M.C. and Martinez-Carrion, M (1983) Biochemistry 22, 3807-3811.

32 Lee, A.G. (1982) in Techniques in the Life Sciences, Lipid and Membrane Biochemistry, B4/II (Hesketh, T.R., Kornberg, H.L., Metcalfe, J.C., Northcote, C.H., Pogson, C.I. and Tipton, K.F., eds.), pp. B422/1-B422/49, Elsevier, Amsterdam.

33 Lechleiter, J., Wells, M. and Gruener, R. (1986) Biochim. Biophys. Acta 856, 640-645.

34 Hille, B. (1977) J. Gen. Physiol. 69, 475-496.

35 Earnest, J.P., Wang, H.H. and McNamee, M.G. (1984) Biochem. Biophys. Res. Commun. 123, 862-868.

36 Earnest, J.P., Limbacher, H.P., McNamee, M.G. and Wang, H.H. (1986) Biochemistry 25, 5809-5818.

37 Blanton, M., McCardy, E., Gallaher, T. and Wang, H.H. (1988) Mol. Pharmacol. 33, 634-642.

38 Horvath, L.I., Arias, H.R., Hideg, K., Hankovszky, H.O., Barrantes, F.J. and Marsh, D. (1990) Biochemistry, in press.

39 Blickenstaff, G.D. and Wang, H.H. (1985) Biophys. J. 47, 261a.

40 Ogden, D.C., Sigelbaum, S.A. and Colquhoun, D. (1981) Nature $289,596-598$

41 Aracava, Y., Ikeda, S.R., Daly, J.W., Brookes, N. and Alburquerque, E.X. (1984) Mol. Pharmacol. 26, 304-313.

42 Ikeda, S.R., Aronstam, R.S., Daly, J.W., Aracava, Y. and Alburquerque, E.X. (1984) Mol. Pharmacol. 26, 293-303.

43 Schreier-Muccillo, S., Marsh, D. and Smith, I.C.P. (1976) Arch. Biochem. Biophys. 172, 1-11.

44 Marsh, D. (1982) in Techniques in the Life Sciences, Lipid and Membrane Biochemistry, B4/II (Hesketh, T.R., Kornberg, H.L., Metcalfe, J.C., Northcote, C.H., Pogson, C.I. and Tipton, K.F., eds.), pp. B426/1-B426/44, Elsevier, Amsterdam.

45 Lehrer, S.S. (1971) Biochemistry 10, 3254-3263.

46 Fernandez, M.S. and Fromherz, P. (1977) J. Phys. Chem. 81, $1755-1761$. 